The final authenticated version is available online at https://dx.doi.org/10.1007/978-3-319-16549-3_42 


\title{
Topology-preserving ordering of the RGB space with an evolutionary algorithm
}

\author{
Francisco Flórez-Revuelta \\ Faculty of Science, Engineering and Computing, Kingston University \\ Penrhyn Road, KT1 2EE, Kingston upon Thames, United Kingdom \\ F.Florez@kingston.ac.uk
}

\begin{abstract}
Mathematical morphology (MM) is broadly used in image processing. MM operators require to establish an order between the values of a set of pixels. This is why MM is basically used with binary and grayscale images. Many works have been focused on extending MM to colour images by mapping a multi-dimensional colour space onto a linear ordered space. However, most of them are not validated in terms of topology preservation but in terms of the results once MM operations are applied. This work presents an evolutionary method to obtain total- and P-orderings of a colour space, i.e. RGB, maximising topology preservation. This approach can be used to order a whole colour space as well as to get a specific ordering for the subset of colours appearing in a particular image. These alternatives improve the results obtained with the orderings usually employed, in both topology preservation and noise reduction.
\end{abstract}

Keywords: Colour ordering, topology preservation, mathematical morphology, evolutionary algorithm, RGB

\section{Introduction}

Mathematical morphology (MM) is used for image processing in different applications: noise filtering, shape simplification, edge detection, skeletonisation, shape analysis, segmentation... MM has two basic operators, erosion and dilation, from which many other morphological operations are derived. These operators rely on an ordering of the pixel values. MM is applied to a set provided with an order and with a supremum and an infimum pertaining to that order. This is the reason why MM has been mainly applied to binary or grayscale images. Extension to multivariate data, as colour images, is not straightforward, because there is not an order relationship among vectors.

Several techniques have been developed to extend MM to colour images, getting partial or total orderings of different colour spaces (RGB, HSI, YIQ, Lab...). In marginal ordering MM operations are applied to each image channel independently, treating it as a grayscale image, combining the partial results to get the final image. This method is not generally accepted because new colours not present in the original image can appear due to this channel-wise filtering. 
Another strategy is to treat the colour at each pixel as a vector. Order is then established by projecting the colour space onto a linear space. For instance, in the case of the RGB space, this projection can be defined as:

$$
\psi:[0,1]^{3} \rightarrow \mathbb{N}, \xi \in[0,1]^{3} \rightarrow \psi(\xi) \in \mathbb{N}
$$

If this projection is an injective function, i.e. $\psi\left(\xi_{1}\right) \neq \psi\left(\xi_{2}\right), \forall \xi_{1} \neq \xi_{2}$, then the ordering is total, otherwise it is partial. There is not a single projection to order the $n$ elements in a colour space, but $n$ ! possibilities, i.e. all the possible permutations of the $n$ colours.

As stated in [1] some of the methods [2,3] aim to create a space-filling curve (SFC), i.e. a continuous curve whose range contains the entire colour space. Although [4] considers that total orderings created with SFC are rather artificial and often lack physical interpretation, [5] studied how different SFC, equivalent to some colour orderings, preserve the topology of colour spaces. Recent surveys on multivariate mathematical morphology can be found in [1], [6].

The concept of topology preservation can be used to analyse how well a colour space is ordered. Topology preservation is a well-known problem in selforganising artificial neural networks as they are usually employed to map a high-dimensional input space into a lower dimensional space. Several measures of topology preservation have been proposed in [7-10]. This approach was used in [11] adapting a growing self-organising network to order the RGB space.

In this paper, a measure of topology preservation is used to determine the best total ordering of a colour space, i.e. RGB. Besides, as calculating a total order is computationally expensive, an evolutionary proposal to obtain a $\mathrm{P}$-ordering is also presented. To the best of my knowledge there is not an evolutionary method that had been proposed for ordering a set of elements in terms of topology preservation. This problem is quite similar to other combinatorial optimisation problems, e.g. the Travelling Salesman Problem. Therefore, representation of individuals, and crossover and mutation operators already employed in those problems will be used in this work.

The remainder of this paper is organised as follows: Section 2 presents a measure of topology preservation and its application to different orderings. In Section 3, an evolutionary method to obtain a partial order of the RGB is presented based on the clustering of the RGB space and the ordering, maximising topology preservation, of the obtained cluster centres. Section 4 shows how these orderings are employed in MM operations. In Section 5 different orderings obtained with this method are shown and compared with state-of-the-art methods. Finally, Section 6 presents some conclusions and future work.

\section{Topology preservation of colour orders}

The topographic product [7] is the most used topology-preservation measure in the area of self-organising neural networks where a low-dimensional network tries to adapt and represent a higher-dimensional input space. The topographic product compares the neighbourhood relationship between each pair of nodes in 
the network with respect to both their position in the network/graph/order and their reference vectors. The topological relationship between a node $j$ and its $k$ closer neighbours is obtained as:

$$
P_{3}(j, k)=\left[\prod_{l=1}^{k} \frac{d^{V}\left(w_{j}, w_{n_{l}^{A}(j)}\right)}{d^{V}\left(w_{j}, w_{n_{l}(j)}\right)} \cdot \frac{d^{A}\left(j, n_{l}^{A}(j)\right)}{d^{A}\left(j, n_{l}^{V}(j)\right)}\right]^{\frac{1}{2 k}}
$$

where $j$ is a node, $w_{j}$ is its reference vector, $n_{l}^{V}$ is the $l$-th closest neighbour to $j$ in the input space $V$ according to a distance $d^{V}$ and $n_{l}^{A}$ is the $l$-th nearest node to $j$ in the network according to a distance $d^{A}$.

This is extended to all the nodes and all the possible neighbourhoods orders, obtaining the topographic product $P$ :

$$
P=\frac{1}{N(N-1)} \sum_{j=1}^{N} \sum_{k=1}^{N} \log \left(P_{3}(j, k)\right)
$$

$P$ is equal to 0 if topology is preserved, deviating from 0 as topology is lost.

Then, the different colour orderings can be measured and compared in terms of their topology preservation of the colour space. Figure 1 shows several of these total orderings as well as their topology preservation value. For the sake of clarity, instead of ordering the entire 3D space, the results are shown for a reduced bidimensional space. The usually employed (a) lexicographical and (b) bit-interlacing orderings preserve worse the topology than other space-filling curves, for instance (c) a Hilbert-type curve with the origin in one corner and the end at the opposite corner [12, Fig. 14]. However, all these orderings are far from (d) the best topology-preserving obtained ordering.

The advantage of the first two methods is that the position of one of the elements in the ordering is easy to estimate and, therefore, obtaining the extrema, i.e. supremum and infimum, in MM operations is fast. Estimating the extrema in orderings that do not follow a "simple" function can be very time consuming. Besides, obtaining the best ordering of a set of elements is an NP-complete problem. In fact, Figure 1d has been obtained using the evolutionary algorithm presented in Section 3, so it cannot be ensured that it is the optimal ordering. Hence, obtaining the best total order for a colour space with a high number of elements, e.g. RGB, is currently infeasible.

\section{Order of the RGB space maximising topology preservation}

This infeasibility suggests that unless the search of the best ordering is reduced to "simple" functions, alternatives should be employed. A P-ordering, as defined in [1], can be obtained by clustering the colour space and ordering the cluster centres. This approach was considered in [11] where a linear growing neural gas was adapted to the RGB space. However, one of the drawbacks of this method is 


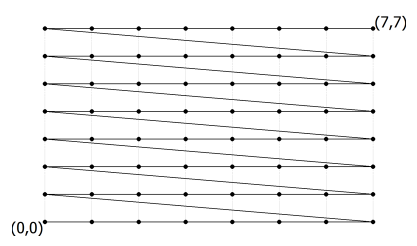

(a) Lexicographical order $|P|=8.34 \times 10^{-2}$

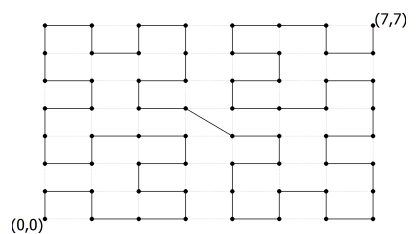

(c) Hilbert space-filling curve $|P|=6.87 \times 10^{-2}$

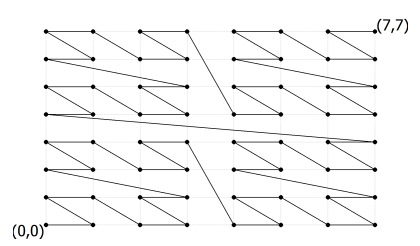

(b) Bit interlacing $|P|=7.60 \times 10^{-2}$

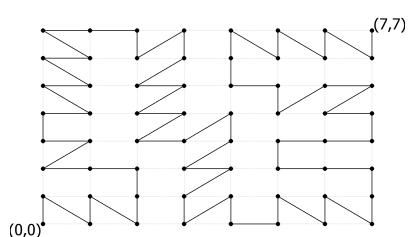

(d) Best obtained order $|P|=6.03 \times 10^{-2}$

Fig. 1: Different orderings of a bidimensional space. Lower $|P|$ values indicate better topology preservation.

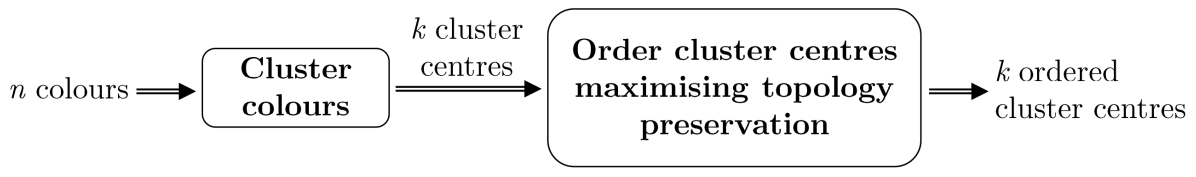

Fig. 2: Scheme of the ordering process.

that both tasks, clustering and ordering, are carried out simultaneously; which affects the final result.

\subsection{Ordering method}

In order to avoid this problem, this paper proposes a two-step process (Figure 2). First, a clustering algorithm is employed to reduce the number of colours to be ordered. There is one constraint in this clustering process: two centres are fixed to $(0,0,0)$ and $(255,255,255)$ respectively. These two centres determine the extrema of the linear ordering. This work employs $k$-means, although any other clustering method can also be applied. Second, these clusters centres are ordered maximising topology preservation.

\subsection{Evolutionary ordering of the cluster centres}

Obtaining the best ordering of a set of elements is an NP-Complete problem, similarly to the Travelling Salesman Problem (TSP). As the number of elements/clusters increases it is more computationally expensive to obtain their 


\begin{tabular}{|c|c|c|c|}
\hline Index & Colour & \multirow{9}{*}{$\begin{array}{c}\text { Chromosome } \\
(1,3,2,5,4,6)\end{array}$} & Order \\
\hline 0 & $(0,0,0)$ & & $(0,0,0)$ \\
\hline 1 & $(59,173,59)$ & & $(59,173,59)$ \\
\hline 2 & $(60,58,175)$ & & $(78,196,196)$ \\
\hline 3 & $(78,196,196)$ & & $(60,58,175)$ \\
\hline 4 & $(177,59,59)$ & & $(195,82,195)$ \\
\hline 5 & $(195,82,195)$ & & $(177,59,59)$ \\
\hline 6 & $(195,197,80)$ & & $(195,197,80)$ \\
\hline 7 & $(255,255,255)$ & & $(255,255,255)$ \\
\hline
\end{tabular}

Fig. 3: Representation of the individual. The pre-established extrema are not considered in the chromosome.

best topology-preserving order. This is the reason why an evolutionary algorithm is proposed, in this paper, to obtain the ordering. The proposal employs a representation of the individuals, and crossover and mutation operators similar to those employed in other combinatorial optimisation problems.

Representation of individuals The proposed evolutionary algorithm employs the well-known path representation where the chromosome represents the sequence that establishes the order of the different colours or cluster centres. However, as the extrema of the ordering, typically $(0,0,0)$ and $(255,255,255)$, are fixed they are not included in the chromosome (Figure 3 ).

Crossover Many different crossover operators have been proposed for combinatorial optimisation. This work employs the Linear Order Crossover (LOX) proposed by Falkenauer and Bouffouix [13]. This operator follows four steps, namely:

1. Select at random a subsequence from one of the parents;

2. Start generating a new offspring by copying that subsequence into the corresponding positions of the new offspring;

3. Delete the elements that are already in the subsequence from the second parent; and

4. Place the elements in the unfilled positions of the offspring from left to right in the order that appear in the second parent.

An example of such a crossover operation is presented in Figure 4.

Mutation Different mutation operators have been proposed for combinatorial optimisation problems[14]. This work makes use of some of them:

- Exchange mutation: selects randomly two elements in the path and exchanges them;

- Swap mutation: selects randomly two consecutive elements and swaps them; 


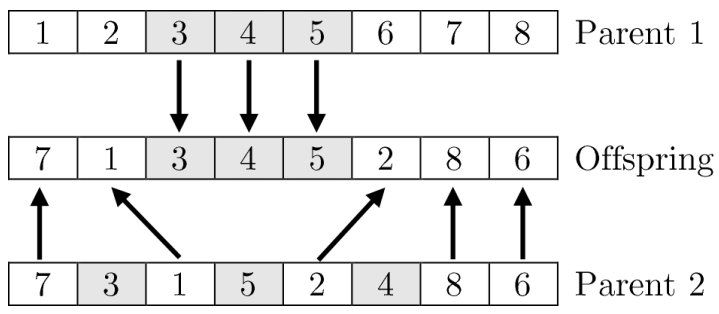

Fig. 4: Application of the Linear Crossover Operator.

- Insertion mutation: selects randomly one element in the path, removes it from the current position, and inserts it in a randomly selected place;

- Simple inversion mutation: selects randomly two elements and it reverses the subsequence between those two cut points;

- Scramble mutation: selects a random subsequence and scrambles the elements in it; and

- Displacement mutation: selects a subsequence at random, removes it from the current position, and inserts it in a random place.

This work also includes a Subsequence ordering mutation that selects a random subsequence and orders it using the same evolutionary algorithm, following a recursive approach. This operator allows a faster convergence of the global evolution as it gets good solutions for a subsequence, with an associated lower computational cost.

\subsection{Ordering of the colours}

Given a pair of colours $\xi_{1}, \xi_{2} \in[0,1]^{3}, \xi_{1}$ is lower than $\xi_{2}$ if $\operatorname{centre}\left(\xi_{1}\right)$ is lower than centre $\left(\xi_{2}\right)$, according to the ordering obtained by the evolutionary process, where $\operatorname{centre}\left(\xi_{i}\right)$ is the nearest cluster centre to colour $\xi_{i}$. However, both colours may be mapped onto the same cluster. In this case, different criteria can be considered to establish an order between them. For instance, ordering them according to their distance to the cluster centre or to their distance to the centre of the structuring element. However, after testing different alternatives, the best results have been obtained by comparing the relationship between the distances from each colour to the neighbouring cluster centres (Figure 5). In that figure, $\xi_{1}<\xi_{2}$ because $\left(d_{\xi_{1}}^{i-1} / d_{\xi_{1}}^{i+1}\right)<\left(d_{\xi_{2}}^{i-1} / d_{\xi_{2}}^{i+1}\right)$. In case that both colours are mapped to the first cluster centre in the ordering then $d_{\xi_{1}}^{i-1}=d_{\xi_{2}}^{i-1}=1$. Similarly, if they are mapped to the last centre, then $d_{\xi_{1}}^{i+1}=d_{\xi_{2}}^{i+1}=1$.

\section{Aplication to mathematical morphology}

Once a colour order $\psi$ has been established, thereby allowing the calculation of the supremum $\left(\sup ^{\psi}\right)$ and the infimum $\left(i n f^{\psi}\right)$ from a set of colours; the 


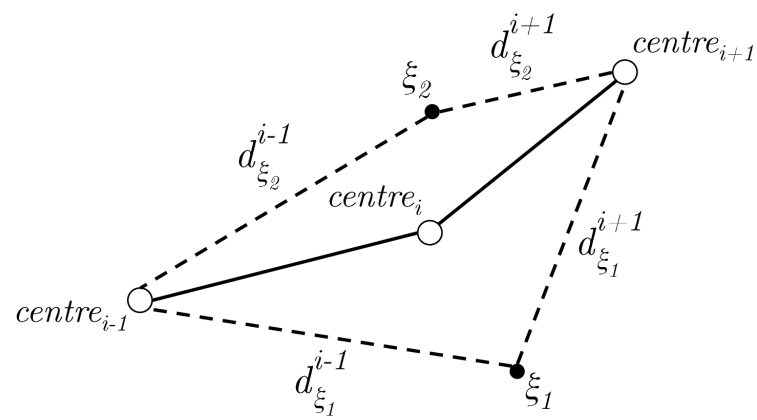

Fig. 5: Comparison between a pair of colours $\xi_{1}$ and $\xi_{2}$ when both are assigned to the same cluster centre centre $_{i}$. centre $_{i-1}$ and centre $_{i+1}$ are its neighbouring clusters centres according to the calculated ordering.

morphological operators for vectorial erosion $\left(\epsilon_{B}^{\psi}\right)$ and dilation $\left(\delta_{B}^{\psi}\right)$ of an RGB image $I$ using structuring element $B$ can be defined at every pixel $(x, y)$ as:

$$
\begin{aligned}
\epsilon_{B}^{\psi}(I)(x, y) & =\inf _{\forall b \in B}^{\psi} I(x+b, y+b) \\
\delta_{B}^{\psi}(I)(x, y) & =\sup _{\forall b \in B}^{\psi} I(x+b, y+b)
\end{aligned}
$$

Structuring element $B$ defines the set of pixels that are considered in each morphological operation. They can have arbitrary shapes, but usually disks, squares, or crosses are employed.

All the other morphological operators using the colour order can be derived from these vectorial erosion and dilation definitions. For instance, the opening $\left(\gamma_{B}^{\psi}\right)$ and the closing $\left(\phi_{B}^{\psi}\right)$ are defined as:

$$
\begin{aligned}
& \gamma_{B}^{\psi}(I)(x, y)=\delta_{B}^{\psi}\left(\epsilon_{B}^{\psi}(I)\right)(x, y) \\
& \phi_{B}^{\psi}(I)(x, y)=\epsilon_{B}^{\psi}\left(\delta_{B}^{\psi}(I)\right)(x, y)
\end{aligned}
$$

\section{Experimentation}

Comparing results with other works has been very difficult as there is not a public dataset that researchers in the field of colour mathematical morphology commonly employ. Most of the works compare the orderings based on the results obtained using them in mathematical morphology operations for specific applications, e.g. noise reduction. This paper follows a similar approach, choosing similar testbeds than the state-of-the-art methods.

The options and parameters of the evolutionary algorithm are: 


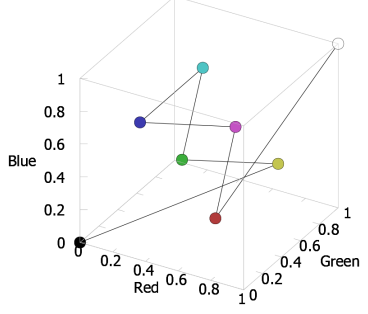

(a) RGB space (8 clusters)

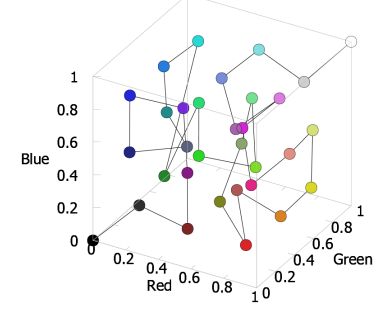

(b) RGB space (32 clusters)

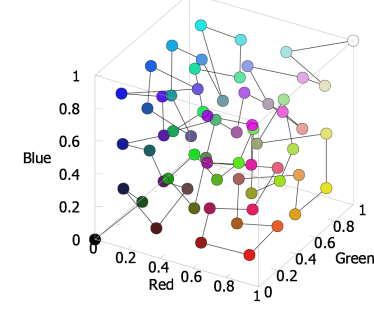

(c) RGB space (64 clusters)

Fig. 6: Evolutionary orderings of the RGB space for different number of cluster centres: (top) ordering in the 3D space, (bottom) ordered sequence of colours.

\begin{tabular}{|c|c|c|c|}
\hline Number of clusters & Lexicographical (RGB) & Bit interlacing & Evolutionary \\
\hline 8 & 0.334 & 0.358 & $\mathbf{0 . 2 1 8}$ \\
\hline 32 & 0.662 & 0.557 & $\mathbf{0 . 4 2 4}$ \\
\hline 64 & 0.721 & 0.549 & $\mathbf{0 . 4 6 3}$ \\
\hline
\end{tabular}

Table 1: Absolute value of the topographic product $|P|$ for the different orderings of the colours shown in Figure 6.

- The size of the population has been set to 25 individuals;

- Parents are selected by fitness-ranking from the current population;

- All the mutations have the same probability to be selected;

- Next generation's population is selected by ranking with elitism for the best; and

- The evolution finishes if the best individual has not changed for 1,000 generations.

As stated above, the application of the Subsequence ordering mutation operator calls a new instance of the evolutionary algorithm with a subsequence chosen at random. In order to avoid a long duration in this recursive evolution the lenght of the subsequence is selected in the interval $[8,24]$. These lengths have been empirically chosen as it has been observed that solutions for shorter subsequences are obtained almost immediately, and it is difficult to get a fast convergence for longer subsequences. Also for a fast convergence, the population has only 10 individuals and the number of generations without changes is limited to 100. Besides, in order to avoid further recursivity, the new EA does not use the Subsequence ordering mutation operator.

The following results and figures employ the best ordering obtained, out of 50 runs, for the set of colours considered in each case.

Results for RGB orderings with different number of clusters are shown in Figure 6 . Table 1 shows the topographic preservation of those orderings and compares the results with the usually employed orderings. Using the topographic 


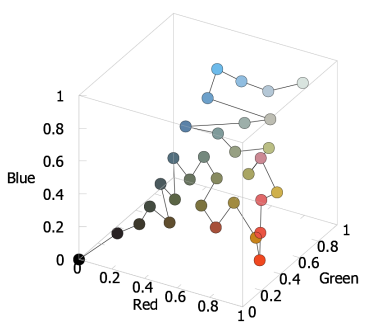

(a) Colours in the Baboon image (32 clusters)

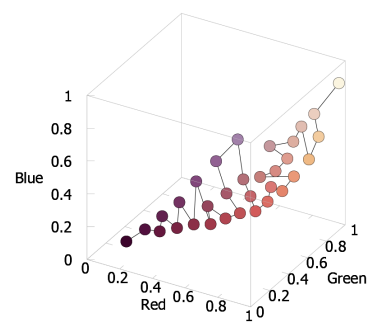

(b) Colours in the Lenna image (32 clusters)

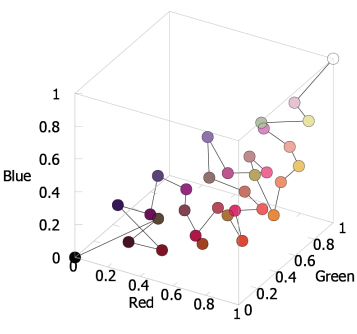

(c) Colours in the noisy Lenna image $(\sigma=32)(32$ clusters)

Fig. 7: Specific orderings for the Baboon and Lenna images (see Figure 8).

product for quality measurement, the evolutionary approach proposed in this paper obtains quite better quantitative results.

Recently, a new approach [15] considers the ordering of the colours that appear in a specific image or a set of them, not in the whole colour space. Then, orderings are specialised, leading to best results in MM operations. Adaptation of the evolutionary proposal to this specific ordering approach requires that the extrema of the ordering are set to the two colours in the image more similar to $(0,0,0)$ and $(255,255,255)$. As fewer colours have to be clustered, the quantisation error of the clustering decreases, obtaining an ordering that should improve the results of later operations (Figure 7).

Following the approach in [1], the orderings proposed in this paper have also been examined in terms of noise reduction quality. The filter employed for smoothing is the open-close close-open (OCCO) operation defined for each pixel $(x, y)$ as:

$$
O C C O_{B}^{\psi}(I)(x, y)=\frac{1}{2}\left(\gamma_{B}^{\psi}\left(\phi_{B}^{\psi}(I)\right)(x, y)+\phi_{B}^{\psi}\left(\gamma_{B}^{\psi}(I)\right)(x, y)\right)
$$

where the structuring element $B$ is a cross of size $3 \times 3$. In all the MM operations the vectorial representation of each colour is mapped into its corresponding position in the ordering.

This operator has been applied to different images corrupted with uncorrelated gaussian noise (Figure 8). The results in terms of normalised mean squared error $(N M S E)$ are shown in Table 2 . The best results are obtained when the specific orders for each one of the images are learned. They are quite stable, improving in almost all the cases the results obtained with the usually employed lexicographical and bit-interlacing orders. They also improve the results presented in [1] and [11]. In the case of a general ordering for the whole RGB space the results are improved as the number of clusters is increased. This is what it should be expected, as in the limit where the number of clusters is equal to the number of colours in the RGB space the best topology-preserving total ordering would be obtained, and noise reduction should be improved. However, this is not 


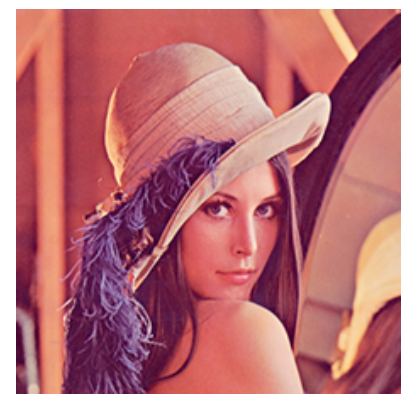

(a) Lenna image

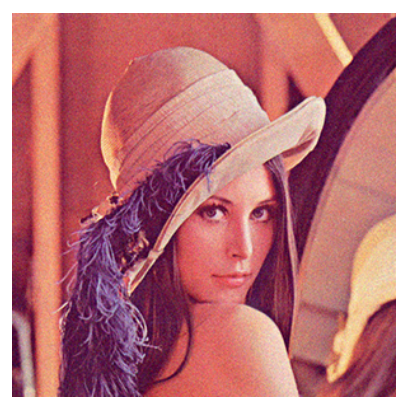

(b) Noisy Lenna image $(\sigma=16)$

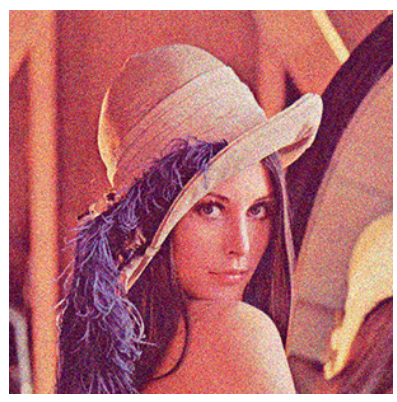

(c) Noisy Lenna image $(\sigma=32)$

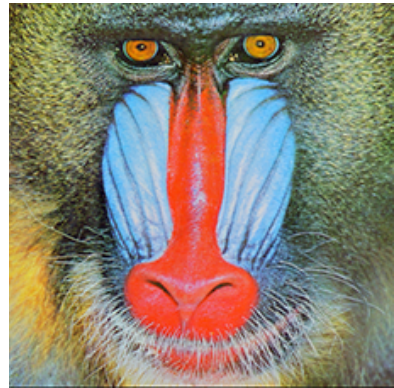

(d) Baboon image

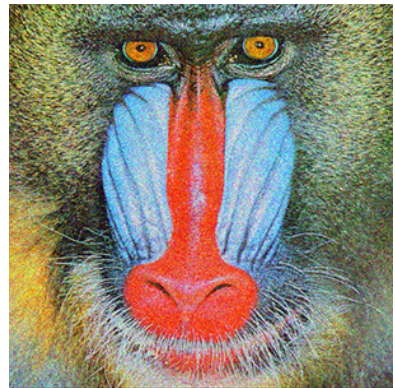

(e) Noisy Baboon image $(\sigma=32)$

Fig. 8: Images used in the experimentation.

the case for the Lenna image $(\sigma=16)$ where NMSE increases with 64 clusters. The explanation to this is that the search space increases and the evolutionary algorithm used for calculating the ordering gets stuck in local minima. This is also the case when using specific orderings. Another cause for an increase in $N M S E$ incrementing the number of clusters in specific orderings is that noisy colours interfere in the calculation of the cluster centres (see in Figures $7 \mathrm{~b}$ and 7c the differences in the positions of the cluster centres because of the noise). Similar tests have been carried out to other images and noise levels, obtaining similar results.

\section{Conclusions}

This paper has presented a method to order the RGB space maximising topology preservation. As calculating topology preservation for the whole colour space is very expensive, a proposal based on clustering the colour space and ordering of the cluster centres has been proposed. This approach has been also employed to obtain specific orders for individual images. Extension to other colour spaces is straightforward modifying how the distances between colours are obtained, e.g. 


\begin{tabular}{|l|c|c|c|c|}
\hline \multirow{2}{*}{ Ordering } & \multirow{2}{*}{$\begin{array}{c}\text { Number of } \\
\text { clusters }\end{array}$} & $\begin{array}{c}\text { Lenna } \\
(\sigma=16)\end{array}$ & $\begin{array}{c}\text { Lenna } \\
(\sigma=32)\end{array}$ & $\begin{array}{c}\text { Baboon } \\
(\sigma=32)\end{array}$ \\
\hline \multirow{3}{*}{ General } & 8 & 1.02 & 2.57 & 5.2 \\
\cline { 2 - 5 } & 32 & 0.81 & 2.16 & 4.46 \\
\cline { 2 - 5 } & 64 & 0.90 & $\mathbf{2 . 0 9}$ & $\mathbf{4 . 2 9}$ \\
\hline \multirow{3}{*}{ Specific } & 8 & $\mathbf{0 . 7 3}$ & $\mathbf{2 . 0 3}$ & 4.38 \\
\cline { 2 - 5 } & 32 & $\mathbf{0 . 7 4}$ & $\mathbf{2 . 0 8}$ & $\mathbf{4 . 3 2}$ \\
\hline Growing Neural & 64 & $\mathbf{0 . 7 5}$ & $\mathbf{2 . 1 0}$ & $\mathbf{4 . 3 3}$ \\
\cline { 2 - 5 } Gas [11] & 3 & 0.84 & 2.23 & 4.50 \\
\cline { 2 - 5 } & 64 & 0.87 & 2.35 & 4.69 \\
\hline Lexicographical & $(\mathrm{RGB})$ & 0.89 & 2.28 & 4.62 \\
\hline Bit interlacing & & 0.75 & 2.16 & 4.35 \\
\hline
\end{tabular}

Table 2: Normalised mean squared error obtained against images corrupted with Gaussian noise. In bold the results that improve the NMSE obtained with the bit-interlacing order.

for the HSI space, in both the clustering and the calculation of the topographic product.

The main drawback of obtaining the best topology-preserving order is its computational cost. This paper has solved this issue proposing an evolutionary algorithm. However, further improvements need to be done in order to obtain better solutions and avoid local minima if the number of clusters is high, i.e. greater than 32 .

Another future work will deal with finding the best "simple" topology-preserving total order for each colour space. Simple understood as easiness to estimate the extrema in MM operations.

Finally, a similar approach is going to be applied to other multivariate data to order higher-dimensional spaces, e.g. histograms, images...thereby allowing the application of mathematical morphology to other multivariate spaces.

\section{References}

1. Aptoula, E., Lefvre, S.: A comparative study on multivariate mathematical morphology. Pattern Recognition 40(11) (2007) 2914-2929

2. Regazzoni, C., Teschioni, A.: A new approach to vector median filtering based on space filling curves. IEEE Transactions on Image Processing 6(7) (Jul 1997) 1025-1037

3. Stringa, E., Teschioni, A., Regazzoni, C.S.: A classical morphological approach to color image filtering based on space filling curves. In: Proceedings of the IEEEEurasip Workshop on Nonlinear Signal and Image Processing, Antalya, Turkey. (1999) 351-354

4. Soille, P., Pesaresi, M.: Advances in mathematical morphology applied to geoscience and remote sensing. IEEE Transactions on Geoscience and Remote Sensing 40(9) (Sep 2002) 2042-2055 
5. Chanussot, J., Lambert, P.: Total ordering based on space filling curves for multivalued morphology (1998)

6. Angulo, J.: Morphological colour operators in totally ordered lattices based on distances: Application to image filtering, enhancement and analysis. Computer Vision and Image Understanding 107(1-2) (2007) 56-73

7. Bauer, H.U., Pawelzik, K.: Quantifying the neighborhood preservation of selforganizing feature maps. IEEE Transactions on Neural Networks 3(4) (1992) 570 579

8. Kaski, S., Lagus, K.: Comparing self-organizing maps. In Malsburg, C., Seelen, W., Vorbrggen, J., Sendhoff, B., eds.: Artificial Neural Networks ICANN 96. Volume 1112 of Lecture Notes in Computer Science. Springer Berlin Heidelberg (1996) 809-814

9. Villmann, T., Der, R., Herrmann, M., Martinetz, T.: Topology preservation in selforganizing feature maps: exact definition and measurement. IEEE Transactions on Neural Networks 8(2) (1997) 256-266

10. Bauer, H.U., Herrmann, M., Villmann, T.: Neural maps and topographic vector quantization. Neural Networks 12(45) (1999) $659-676$

11. Flórez-Revuelta, F.: Ordering of the RGB Space with a Growing Self-organizing Network. Application to Color Mathematical Morphology. In Duch, W., Kacprzyk, J., Oja, E., Zadrony, S., eds.: Artificial Neural Networks: Biological Inspirations ICANN 2005. Volume 3696 of Lecture Notes in Computer Science. Springer Berlin Heidelberg (2005) 385-390

12. Rose, N.: Hilbert-type space-filling curves. http://www4.ncsu.edu/ njrose/ pdfFiles/HilbertCurve.pdf (2001)

13. Falkenauer, E., Bouffouix, S.: A genetic algorithm for job shop. In: Proceedings of the IEEE International Conference on Robotics and Automation, 1991. (Apr 1991) 824-829 vol.1

14. Larrañaga, P., Kuijpers, C.M.H., Murga, R.H., Inza, I., Dizdarevic, S.: Genetic algorithms for the travelling salesman problem: A review of representations and operators. Artificial Intelligence Review 13(2) (1999) 129-170

15. Chevalier, E., Angulo, J.: Image adapted total ordering for mathematical morphology on multivariate images. In: Proceedings of the IEEE International Conference on Image Processing. (2014) 2943-2947 